\title{
Éditorial
}

\section{Alain Béry}

SQODF - Maître de conférences honoraire - Administrateur de la FFO - Directeur de publication de la Revue d'ODF

\section{QUAND LA NOUVEAUTÉ DEVIENT-ELLE UNE DONNÉE AVÉRÉE DE LA SCIENCE ?}

Code de la santé publique

Art. R. 4127-32. (D. n²004-802, 29 juill. 2004, art. 1 $^{\mathrm{er}}$ )

Dès lors qu'il a accepté de répondre à une demande, le médecin s'engage à assurer personnellement au patient des soins consciencieux, dévoués et fondés sur les données acquises de la science, en faisant appel, s’il y a lieu, à l'aide de tiers compétents.

Art. R. 4127-233.- (D. $n^{\circ}$ 2004-802, 29 juill. 2004, art. $1^{\mathrm{er}}$ )

Le chirurgien-dentiste qui a accepté de donner des soins à un patient s'oblige :

$1^{\circ}$ A lui assurer des soins éclairés et conformes aux données acquises de la science, soit personnellement, soit lorsque sa conscience le lui commande en faisant appel à un autre chirurgien-dentiste ou à un médecin...

\section{Arrêt Mercier}

Selon l'analyse classique, la relation juridique médecin/patient est de nature contractuelle. Le principe a été posé par l'arrêt Mercier, de la Cour de cassation, en date du 20 mai 1936.

Par cet arrêt, la Cour de cassation a posé en principe « qu'il se forme entre le médecin et son client, un véritable contrat, comportant pour le praticien l'engagement, sinon bien évidemment de guérir le malade, ce qui n'a d'ailleurs jamais été allégué, du moins de lui donner des soins, non pas quelconques ainsi que paraît l'énoncer le moyen du pourvoi mais consciencieux, attentifs et, réserve faite de circonstances exceptionnelles, conformes aux données acquises de la science. »

\section{Données actuelles}

L'arrêt Mercier faisait référence aux données acquises de la science. Certaines décisions postérieures visaient les données actuelles de la science. La doctrine s'était interrogée sur la portée de ce changement de terminologie. Certains auteurs le critiquaient, craignant que le juge soit tenu de s'intéresser de plus près à l'évolution des données scientifiques. D’autres auteurs considéraient simplement que la formule nouvelle est plus conforme au 
devoir d'entretien des connaissances imposées par l'article 16 du code de déontologie (devenu Art. R. 4127-214 du Code de la Santé publique).

Mais la Cour de cassation a mis un terme à ce débat, en condamnant très clairement la nouvelle terminologie et en consacrant à nouveau la référence aux données acquises de la science (Cass. ${ }^{\text {re }}$ civ, 6 juin 2000). Mais attendu, d'abord, que l'obligation pesant sur un médecin est de donner à son patient des soins conformes aux données acquises de la science à la date de ces soins ; que la troisième branche du moyen, qui se réfère à la notion, erronée, de données actuelles est dès lors inopérante ;

\section{Données de la science}

Les données de la science sont les données connues, ayant fait l'objet de publication. De plus, elles doivent avoir reçu l'assentiment de la partie la plus considérable de la communauté scientifique.

\section{Publications}

Selon la jurisprudence dominante, il s'agit des données connues, ayant fait l'objet de publications : le médecin n'est pas responsable de la nécrose ischémique des muscles du mollet provoquée par une forme rare et malencontreuse de la fracture subie par le patient, dès lors qu'à l'époque des faits aucune publication n'avait eu lieu en France sur cette question (Cass. $1^{\text {re }}$ civ, 12 nov. 1985, no 83-17.061).

\section{Communauté scientifique}

En outre, ces données doivent avoir reçu l'assentiment de la partie la plus considérable de la communauté scientifique : on ne peut reprocher à un chirurgien l'omission d'une ponction crico-thyroïdienne, dès lors que cette opération, à l'époque des faits, avait été décrite dans la littérature médicale, mais n'était pas encore de pratique courante (Cass. crim. 3 nov. 1988). Les références jurisprudentielles peuvent paraître surannées voire obsolètes, mais il n'y a pas eu de jugement plus récent ; elles font donc toujours autorité.

\section{Les recommandations de la HAS}

Si la référence aux données acquises de la science constitue toujours le critère d'appréciation de la faute médicale, on peut toutefois se demander quelle sera l'incidence, sur ce point, des références médicales opposables instituées par la convention médicale de 1993 et si elles sont appelées à se substituer aux données de la science pour juger de la responsabilité des praticiens.

La section des assurances sociales du Conseil national de l'ordre des médecins, a condamné pour la première fois, en 2003, un médecin gynécologue à quatre mois d'interdiction pour n'avoir pas suivi une recommandation de bonnes pratiques élaborées par l'ANAES, devenue la Haute Autorité de santé (HAS) depuis la loi du 13 août 2004. Le Conseil d'État, saisi par le médecin sanctionné en annulation de cette décision, a rejeté la requête en considérant que la section des assurances du Conseil national de l'ordre des médecins n'avait pas commis d'erreur de droit et qu'elle avait pu estimer que le médecin requérant «n'avait pas tenu compte pour dispenser ses soins à ses patients des données acquises de la science, telles qu'elles résultent notamment des recommandations de bonnes pratiques élaborées par l'Agence nationale d'évaluation et d'accréditation... » 
Non respect des données de la science

Le seul fait de poser un diagnostic erroné ne constitue pas, en soi, une faute médicale. Les juges ne peuvent donc condamner un médecin pour erreur de diagnostic sans se prononcer sur la question de la difficulté ou non de poser le bon diagnostic dans la situation concrète qui leur est soumise (Cass. $1^{\text {re }}$ civ, 31 mai 2007).

L'erreur de diagnostic ne devient fautive que lorsqu'elle résulte d'une méconnaissance par le médecin de ses obligations, soit parce qu'il n'a pas fait les investigations et explorations nécessaires, soit parce qu'il n'a pas su interpréter conformément aux données de la science les symptômes traduisant l'état interne du malade.

\section{Traitement tombé en désuétude}

Le praticien peut également recourir à un traitement tombé en désuétude (CA Paris, $1^{\text {re }}$ ch., 3 mars 1972,) ou, à l'inverse, préconiser un traitement peu usuel (CA Toulouse, 26 mai 1939; CA Paris, 26 avr. 1984).

Cette liberté est, bien entendu, tempérée par la nécessité de se conformer aux usages et de respecter les données acquises de la science. En conséquence, le recours à un traitement en désuétude devient fautif lorsque ce traitement a été abandonné en raison de sa dangerosité.

A. Béry

Les opinions émises n'engagent que leurs auteurs. 